\section{B. PICARD, \\ M.-P. DURIS, C. JURIE}

INRA Laboratoire

Croissance et Métabolismes des Herbivores, Theix, 63122 Saint-Genès Champanelle

\title{
Caractérisation des chaînes lourdes de myosine dans le muscle de bovin
}

Les études concernant les qualités organoleptiques de la viande bovine se focalisent sur la tendreté car c'est le critère le plus important pour le consommateur. Parmi les facteurs déterminant cette tendreté, le type de fibres joue un grand rôle. En effet, la vitesse de maturation dépend de la composition en fibres (Valin 1988). Actuellement, la tendreté de la viande présente une grande variabilité qui est la conséquence de la diversité de l'élevage bovin français. Différentes études ont en effet montré que les conditions d'élevage (âge, sexe, race, niveau énergétique et nature de la ration...) modifient la composition en fibres des muscles (Geay et Renand 1994). Aussi, l'objectif des recherches sur la croissance musculaire des bovins est de décrire la mise en place des fibres durant le stade fotal et de préciser l'influence des différents facteurs sur la composition en fibres des muscles durant la vie post-natale. Ces études nécessitent une méthode fiable pour classer les différents types de fibres. Les techniques d'immunohistochimie, de Western-blot et de dosage ELISA sont couramment utilisées et ont permis d'obtenir des résultats importants (Picard et al 1994 et 1995). Cependant, les anticorps disponibles dans le commerce ne permettent pas d'identifier spécifiquement les fibres IIA et IIB. Il apparaît indispensable de disposer d'anticorps pour suivre la différenciation et l'évolution de ces fibres. Cet article fait le point sur les possibilités qu'offrent les anticorps obtenus dans le projet Noé concernant ces applications.

\section{Caractérisation des anticorps}

Les 50 anticorps retenus après un premier tri ont été testés en immunohistologie. Pour cela des coupes sériées transversales de $10 \mu \mathrm{m}$ d'épaisseur ont été mises en contact pendant 30 minutes avec le premier anticorps (surnageant pur), rincées avec du tampon phosphate, puis incubées pendant 30 minutes avec le second anticorps fluorescent (lapin anti-IgG de souris couplé au FITC, Jackson Immunotech) dilué au 1/30 dans du tampon phosphate. Les coupes ont été lavées avec le tampon phosphate et montées entre lame et lamelle avec du mowiol. A l'issue de ce testage sur des échantillons de 3 muscles de bovin adulte (Cutaneus trunci : MHC IIa et IIb ; Diaphragma : MHC I et IIa ; Semitendinosus MHC I, IIa et IIb) et sur le muscle Longissimus dorsi de fotus bovin (âgé de 210 jours), une dizaine d'anticorps a été retenue. Très rapidement, quelques anticorps retestés avaient perdu de leur réactivité et ont été abandonnés. En définitive, 4 anticorps ont été retenus. L'intensité de leur réponse en immunohistologie est décrite dans le tableau 1.

Les 2 anticorps monoclonaux S5 8H2 et S5 $15 \mathrm{~F} 4$ ont également été testés en Western, en ELISA et en culture de cellules. Leur réponse suite à ces différentes techniques est positive et cohérente avec la réponse obtenue en immunohistologie.

S5 8 H2 est un très bon anticorps tant pour l'intensité de la réponse que pour l'informa-

Tableau 1. Réactivité des anticorps monoclonaux anti-chaine lourde de myosine avec les différents types de fibres $(I, I I A, I I B)$ dans le muscle de bovin. L'intensité de la réponse est notée : +++ très forte ; ++ forte ; + faible ; - négative ; non testé.

\begin{tabular}{|c|c|c|c|c|}
\hline Anticorps & S5 8H2 & S5 15F4 & S4 10B6 & S5 7E6 \\
\hline \multicolumn{5}{|l|}{ Muscle de bovin adulte } \\
\hline Cutaneus trunci & & & & \\
\hline IIA & - & +++ & - & + \\
\hline IIB & +++ & +++ & ++ & + \\
\hline \multicolumn{5}{|l|}{ Diaphragma } \\
\hline I & +++ & - & - & + \\
\hline IIA & - & +++ & - & + \\
\hline \multicolumn{5}{|l|}{ Semitendinosus } \\
\hline I & ++ & - & - & + \\
\hline IIA & - & +++ & - & + \\
\hline IIB & ++ & +++ & ++ & + \\
\hline \multirow{3}{*}{\multicolumn{5}{|c|}{$\begin{array}{l}\text { Muscle de fœtus } \\
\text { bovin culard ( } 210 \mathrm{j} \text { ) } \\
\text { Longissimus dorsi }\end{array}$}} \\
\hline & & & & \\
\hline & & & & \\
\hline $\mathrm{I}$ & ++ & - & 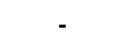 & $?$ \\
\hline II & ++ & ++ & ++ & $?$ \\
\hline Spécificité & anti-I+IIlb & anti-II & anti-IIb & anti-myosine \\
\hline
\end{tabular}


tion qu'il donne. Il est à la fois anti-MHC I et anti-MHC IIb (figure 1). Il permet la classification des fibres I et des fibres IIB et donc des fibres IIA par différence. Il répond ainsi à l'objectif de départ : différencier les fibres IIA et IIB. Il permet la distinction de 2 populations de fibres rapides chez le fotus dès 260 jours de gestation. Il peut être utilisé en immunohistologie, en Western-blot et en culture de cellules, aussi bien chez le fotus que chez l'adulte. Par contre, il ne peut pas être utilisé en ELISA bien qu'il donne une réponse, car il n'existe pas de muscle témoin entièrement MHC I + IIb permettant de réaliser une gamme étalon.

S5 15F4 est un très bon anticorps antiMHC rapides (MHC IIa et IIb) (figure 1) qui peut être utilisé aussi bien en immunohistologie (chez le fotus et l'adulte), qu'en ELISA, en Western et également en culture de cellules. De ce point de vue en effet, S5 15F4 est d'un intérêt tout particulier car l'anticorps antiMHC rapides F113 15F4 commercialisé par Biocytex ne donne aucune réponse en culture. De plus, alors que chez l'adulte ils reconnaissent les mêmes cellules, ces deux anticorps anti-myosines rapides donnent des résultats différents au stade fœetal : S5 15F4 reconnaît des cellules qui jusqu'à présent n'étaient reconnues par aucun autre anticorps.

S4 10B6, anticorps anti-MHC IIb (figure 1), fait partie des hybridomes qui ont perdu rapidement de leur réactivité, mais qui donnaient lors des premiers essais de très bons résultats en immunohistologie, en ELISA et en culture. En réalité cet hybridome est très peu concentré et mérite d'être recloné.

S5 7E6 est un anti-myosine totale qui pourrait être utilisé en vue d'un dosage quantitatif de la myosine totale dans différents muscles.

\section{Conclusion}

L'utilisation des anticorps S5 8H2 (antiI+IIb) et S4 10B6 (anti-IIb) permettra de distinguer les fibres IIA des fibres IIB et donc de préciser les différentes étapes de mise en place de ces fibres musculaires durant la vie fœtale ainsi que leur évolution avec l'âge et sous l'influence des différents facteurs d'élevage. De plus, il a déjà été possible, grâce à l'anticorps S5 8H2 (anti-I+IIb) de mettre en évidence une population importante de fibres hybrides, appelées IIAB, représentant environ $10 \%$ des fibres totales (Picard et al 1998). L'étude de ces fibres est un point important pour comprendre comment se produisent les changements de types de fibres. D'autre part, S5 8H2 a également été retenu pour d'autres espèces, en particulier l'espèce porcine. Pour cette espèce il est anti-I+IIb+IIx. Bien que l'isoforme MHC IIx n'ait pas pu être encore identifiée chez le bovin, plusieurs arguments nous amènent à supposer son existence. Selon Hämälaïnen et Pette (1995), dans les muscles squelettiques de mammifères, plus la taille de l'animal est importante, plus la concentration
Figure 1. Révélation immunohistologique des chaînes lourdes de myosine dans le muscle Semitendinosus de bovin à l'aide des anticorps issus du projet Noé.

Anti MHC 1 (5B9 Biocytex)
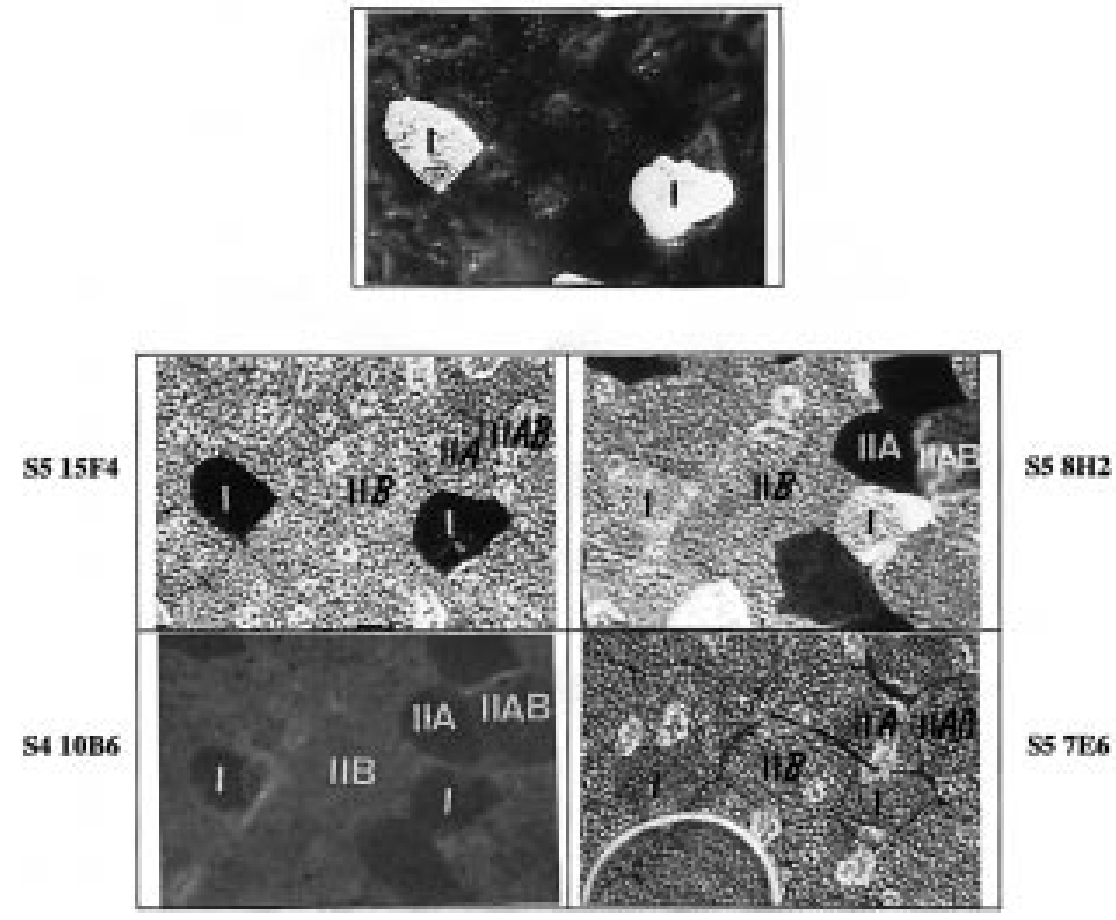

de l'isoforme MHC IIx est élevée et inversement pour MHC IIb. De plus, MHC IIx a un épitope commun avec MHC IIb (Schiaffino et al 1989), ce qui pourrait expliquer que jusqu'à présent ces deux isoformes aient été confondues et laisse supposer que $\mathrm{S} 5 \mathrm{8H} 2$ serait également anti-I+IIb+IIx chez le bovin avec une proportion très importante de MHC IIx dans les muscles de bovin. Enfin, parmi les différents types de fibres présents à 210 jours de vie fœtale, un type n'était reconnu par aucun anticorps parmi ceux existants jusqu'à présent, ceci dans tous les muscles de bovin culard et dans le Cutaneus trunci de bovin non culard (Picard et al 1995). Les anticorps S5 8H2 et S5 15F4 marquent ces cellules qui ne sont reconnues par aucun autre anticorps ; ils apportent donc une information supplémentaire quant à la caractérisation de ces fibres inconnues.

Les différents anticorps retenus dans ce projet permettent donc de nouvelles applications pour l'étude du muscle de bovin. Cependant l'objectif initial, à savoir l'obtention d'anticorps spécifiques des MHC IIa et IIb utilisables en particulier en dosage ELISA n'a pas été atteint et demeure un besoin important.

\section{Références}

Geay Y., Renand G., 1994. Importance de la variabilité génétique et du mode d'élevage des bovins sur les caractéristiques musculaires et les qualités organoleptiques de leur viande. $1^{\text {res }}$ Rencontres Recherches Ruminants, 177-182. 
Hamalaïnen X., Pette X., 1995. Patterns of myosin isoforms in mammalian skeletal muscle fibres. Microsc. Res. Tech., 30, 381-9.

Picard B., Robelin J., Pons F., Geay Y., 1994. Comparison of the foetal development of fibre types in four bovine muscles. J. Muscle Res. Cell Motil., 15, 473486.

Picard B., Gagnière H., Robelin J., Pons F., Geay Y., 1995. Presence of an unidentified myosin isoform in certain bovine foetal muscles. Meat Sci., 41, 315-324.
Picard B., Duris M.-P., Jurie C., 1998. Classification of bovine muscle fibers by different histochemical techniques. Histochem. J., à paraître.

Schiaffino S., Gorza L., Satore S., Saggin L., Ausoni S., Vianello M., Gundersen K., Lomo T., 1989 Three myosin heavy chain isoforms in mammalian skeletal muscle. J. Muscle Res. Cell Motil., 10, 197-205.

Valin C., 1988. Différenciation du tissu musculaire. Conséquences technologiques pour la filière viande. Reprod. Nutr. Dev., 28, 845-856.

\section{LEFAUCHEUR, P. ECOLAN \\ INRA Station de \\ Composition en chaînes lourdes de myosine des fibres musculaires de type II chez le porc}

Recherches Porcines, 35590 Saint-Gilles
Les trois types de fibres musculaires classées I, IIA et IIB par les techniques histoenzymatiques existent dans les muscles squelet-
Figure 1. Coupes transversales sériées de muscle longissimus dorsi chez un porc de $100 \mathrm{~kg}$ de poids vif.

A) ATPase après préincubation à $\mathrm{pH} 4,35$. B) Révélation de l'activité de la succinodéshydrogénase. C-F) Détection histoimmunologique des MHC à l'aide d'anticorps issus du projet Noé. Les chiffres 1, 2 et 3 repèrent 3 fibres homologues sur les différentes coupes sériées. Barre d'échelle : $50 \mathrm{~mm}$.
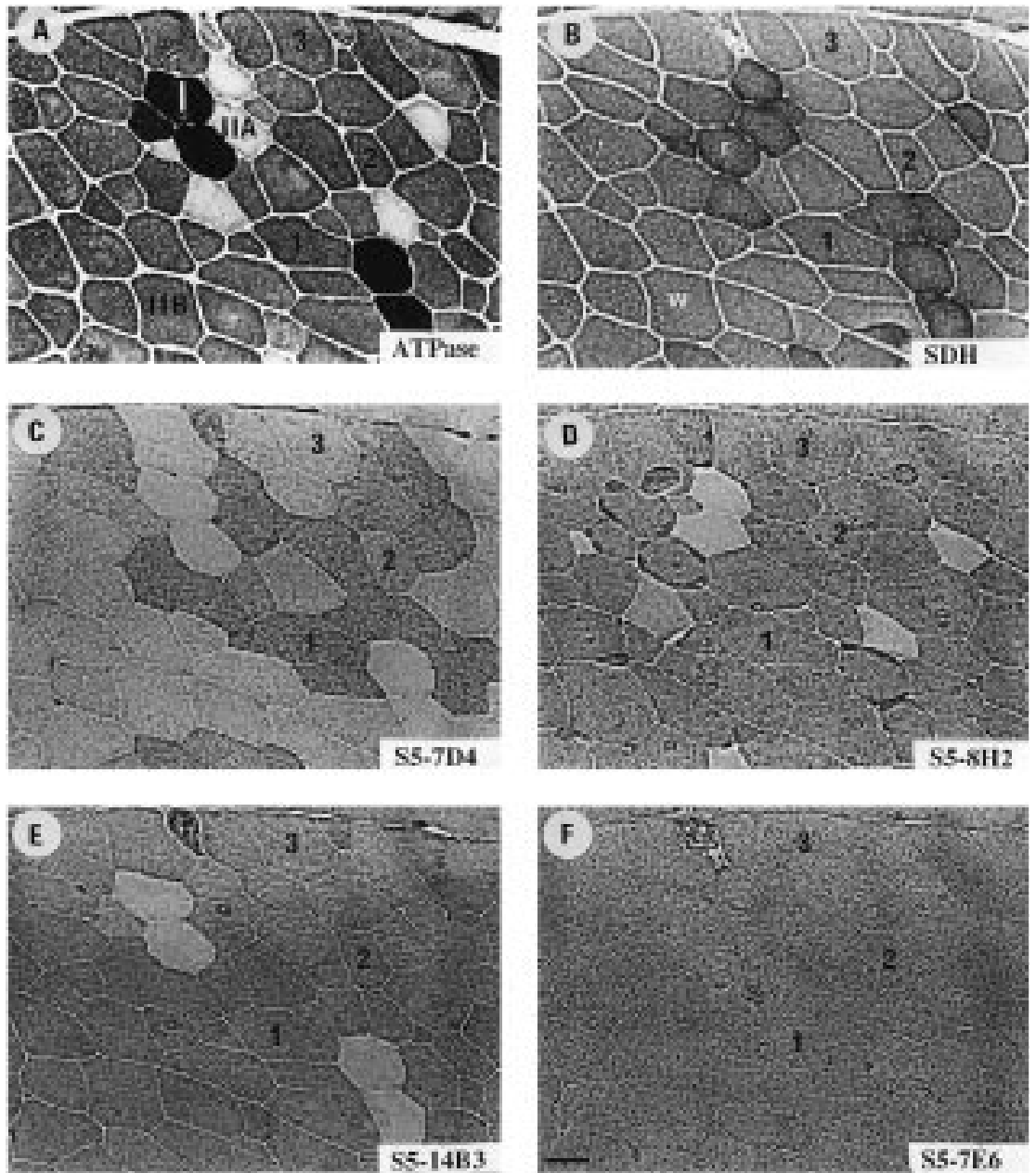

tiques du porc au poids commercial d'abattage (Lefaucheur et al 1991). Cette typologie repose sur un polymorphisme des chaînes lourdes de la myosine (MHC) (Schiaffino et Reggiani 1996). Chez diverses espèces de laboratoire, quatre MHC ont été identifiées dans le muscle squelettique adulte : I, IIa, IIx et IIb (Bär et Pette 1989, Schiaffino et al 1989, DeNardi et al 1993). Ces auteurs ont ainsi montré que les fibres classées IIB lorsqu'elles sont typées par la méthode ATPase constituent une population hétérogène en regard de leur composition en MHC. A notre connaissance, la caractérisation du polymorphisme des MHC de type II n'a pas été réalisée chez le porc, notamment en raison de l'absence d'anticorps spécifiques de chacune des isoformes de MHC. Ce texte rapporte les résultats d'histoimmunologie obtenus avec les anticorps anti-MHC sélectionnés dans le projet Noé.

\section{Conditions expérimentales}

L'étude a été réalisée sur le muscle longissimus dorsi (LD) et la partie rouge du muscle semitendinosus (STR) chez des porcs femelles de race Large White abattus à $100 \mathrm{~kg}$ de poids vif (165 jours d'âge). Les échantillons de muscle ont été prélevés environ 30 minutes après l'abattage, congelés par immersion dans de l'isopentane refroidi par de l'azote liquide et stockés à - $80{ }^{\circ} \mathrm{C}$ jusqu'aux analyses histologiques.

Des coupes transversales sériées de $10 \mu \mathrm{m}$ d'épaisseur ont été réalisées à l'aide d'un microtome à congélation $\left(-20{ }^{\circ} \mathrm{C}\right)$. La coloration ATPase après une préincubation de la coupe à $\mathrm{pH} 4,35$ a permis d'identifier les fibres de types I, IIA et IIB (Brooke et Kaiser 1970). L'activité de la succinodéshydrogénase (SDH) est révélée sur une coupe sériée (Nachlas et al 1957), elle permet de distinguer des fibres rouges $(r)$, intermédiaires (i) et blanches (w) (figure 1B). Les colorations histoimmunolo- 